\title{
Effect of sources on the inner horizon of black holes
}

\author{
Ozay Gurtug* and Mustafa Halilsoy \\ Department of Physics, Eastern Mediterranean University, G.Magusa, North Cyprus, Mersin 10 - Turkey
}

(Received 7 May 2001; published 26 September 2001)

\begin{abstract}
A single pulse of null dust and colliding null dust both transform a regular horizon into a spacelike singularity in the space of colliding waves. The local isometry between such space-times and black holes extrapolates these results to the realm of black holes. However, the inclusion of particular scalar fields instead of null dust creates null singularities rather than spacelike ones on the inner horizons of black holes.
\end{abstract}

DOI: 10.1103/PhysRevD.64.084023

PACS number(s): 04.20.Cv, 04.20.Dw

\section{INTRODUCTION}

In this paper we show, with exact solutions, that the Cauchy horizon $(\mathrm{CH})$ has an indeterminate character with respect to different perturbing potentials and in some cases the $\mathrm{CH}$ can be spacelike singular and in others it can be null singular. The first signs of this sort of indeterminacy of character for the $\mathrm{CH}$ were seen in the outcome of Chandrasekhar and Xanthopoulos (CX) [1] and Yurtsever's [2] analysis of the stability of the horizon (or quasiregular singularity) of the Bell-Szekeres (BS) [3] space-time. These authors used perturbation methods in their work. In Ref. [4] it has been shown that there is a similar inner horizon instability for black holes (BHs) and the horizons change to spacelike singularities. On the other hand, Ori [5] found the horizon of a Kerr $\mathrm{BH}$ changes to a null singularity. All of these works used perturbation methods.

This lack of consensus for the instability of colliding plane waves (CPW) and $\mathrm{BH}$ horizons has attracted much interest and effort. Burko $[6,7]$ confirmed Ori's findings of a regular horizon changing to a null singularity when he applied a scalar field to a Reissner-Nordström (RN) BH. His work was done using numerical methods.

As an alternative to Burko's approach we have applied the local isometry between CPW and the region between the two (event and Cauchy) horizons of BHs. This enables us to couple scalar and other fields to CPWs, where there are analytically tractable solutions, and then to transform our results to BH cases. This approach was first introduced by Yurtsever [8]. Yurtsever concluded that the instabilities of the CHs in Kerr and $\mathrm{RN}$ BHs turns the $\mathrm{CH}$ to a spacelike curvature singularity. Yurtsever's comments were indications of certain possibilities but they remained unsupported.

We exploit the isometry analogy and we consider two sorts of sources in the CX CPW [9] space-time. The first is null propagating dust (with their mutual collision). We concentrate on the CX space-time because it has a nonsingular horizon and it is locally isometric to the Kerr-Newman (KN) $\mathrm{BH}$, enabling us to transform through isometry to the $\mathrm{BH}$ space-time. We find that propagation (or collision) of null dusts in the CX space-time convert the inner horizon to a spacelike singularity. This conclusion is supported by CX [10] for the Einstein-vacuum problem which is locally iso-

\footnotetext{
*Email address: ozay.gurtug@emu.edu.tr
}

metric to the Kerr BH. CX's solution supports our conclusion applied to the $\mathrm{KN} \mathrm{BH}$, because the presence of charge is trivial. We point out that the introduction of coupling in our case is entirely different from the one in CX.

The second is a scalar field in between the horizons. We show that such a field effects the inner horizon differently from the above case and the singularity it creates turns out to be null. In other words our two sorts of coupling show the inner horizon of a BH does not have a unique character in its singularity structure and this character depends on the perturbing potential. The instabilities of these $\mathrm{CHs}$ occurring in CPW space-times and those of corresponding BH spacetimes have dual character.

We also make some comments about the HelliwellKonkowski (HK) [11] conjecture. HK conjecture was thought to enable us to predict the instability of a horizon and the sort of singularity it changes to. However, our conclusion that inner horizons have dual character shows that HK conjecture cannot uniquely determine the sort of the outcoming singularity, hence it should be used with caution.

This paper is organized as follows. In Sec. II we review the connection between the CX and the KN space-times. In Sec. III we consider null dust as a test field in the CX spacetime. Section IV follows with an exact back-reaction solution to the foregoing section. Section $\mathrm{V}$ exposes the role of scalar fields leading to the null singularities in the $\mathrm{KN} \mathrm{BH}$ spacetime. We conclude the paper with a discussion in Sec. VI.

\section{CHANDRASEKHAR-XANTHOPOULOS AND KERR-NEWMAN METRICS}

CX have found a colliding wave solution in the EinsteinMaxwell (EM) theory which is locally isometric to the $\mathrm{KN}$ $\mathrm{BH}$ solution [9]. CX metric is given by

$$
d s^{2}=X\left(\frac{d \tau^{2}}{\Delta}-\frac{d \sigma^{2}}{\delta}\right)-\Delta \delta \frac{X}{Y} d y^{2}-\frac{Y}{X}\left(d x-q_{2 e} d y\right)^{2},
$$

where the coordinates $(\tau, \sigma)$ are given in terms of the null coordinates $(u, v)$ by

$$
\begin{gathered}
\tau=u \sqrt{1-v^{2}}+v \sqrt{1-u^{2}}, \\
\sigma=u \sqrt{1-v^{2}}-v \sqrt{1-u^{2}},
\end{gathered}
$$

and $\Delta=1-\tau^{2}, \delta=1-\sigma^{2}$. 
The metric functions are

$$
\begin{aligned}
X & =\frac{1}{\alpha^{2}}\left[(1-\alpha p \tau)^{2}+\alpha^{2} q^{2} \sigma^{2}\right], \\
Y & =1-p^{2} \tau^{2}-q^{2} \sigma^{2}, \\
q_{2 e} & =-\frac{q \delta}{p \alpha^{2}} \frac{1+\alpha^{2}-2 \alpha p \tau}{1-p^{2} \tau^{2}-q^{2} \sigma^{2}}
\end{aligned}
$$

in which the constants $\alpha, p$, and $q$ must satisfy

$$
\begin{gathered}
0<\alpha \leqslant 1, \\
p^{2}+q^{2}=1 .
\end{gathered}
$$

The metric (1) transforms into the Boyer-Lindquist form of the $\mathrm{KN}$, if the following transformation is used:

$$
t=\max , \quad y=p \phi, \quad \tau=\frac{m-r}{\sqrt{m^{2}-\alpha^{2}-Q^{2}}}, \quad \sigma=\cos \theta
$$

with

$$
p=\frac{\sqrt{m^{2}-a^{2}-Q^{2}}}{m \alpha}, \quad q=-\frac{a}{m \alpha}, \quad\left(m^{2}>a^{2}+Q^{2}\right)
$$

so that $Q^{2}=\left(1-\alpha^{2}\right) m^{2}$ holds. Note that $\alpha=1$ removes the charge and reduces the problem from $\mathrm{KN}$ to Kerr and in particular the limit $a=0$ yields the Reissner-Nördström case. With these substitutions the line element (1) may be written in the form

$$
\begin{aligned}
\alpha^{2} m^{2} d s^{2}= & \left(1-\rho^{-2} \omega\right) d t^{2} \\
& -\sin ^{2} \theta\left[\Delta+\omega\left(1+a^{2} \rho^{-2} \sin ^{2} \theta\right)\right] d \phi^{2} \\
& -2 a \omega \rho^{-2} \sin ^{2} \theta d t d \phi-\rho^{2}\left(\Delta^{-1} d r^{2}+d \theta^{2}\right)
\end{aligned}
$$

with the standard notation

$$
\begin{aligned}
\Delta & =r^{2}-2 m r+a^{2}+Q^{2} \equiv\left(r-r_{-}\right)\left(r-r_{+}\right), \\
\rho^{2} & =r^{2}+a^{2} \cos ^{2} \theta, \\
\omega & =2 m r-Q^{2},
\end{aligned}
$$

in which $a$ and $Q$ stand, respectively, for the constants of rotation and electric charge. Note that $\Delta$ here is different from the $\Delta$ of the CX metric. The roots of $\Delta, r_{+}$, and $r_{-}$are known as the event and Cauchy (inner) horizon, respectively. Therefore the colliding wave solution due to $\mathrm{CX}$ is locally isometric to the $\mathrm{KN}$ metric in between the two horizons.

\section{TEST NULL DUST IN THE CX SPACETIME}

We consider now two null test dusts moving in opposite directions in the interaction region of the $\mathrm{CX}$ metric or equivalently null fields moving in the isometric region of the KN space-time. Such null dusts (and the following exact solution) suffice to expose the nonlinear effect of the background as well as the disturbance of the background (i.e., the back reaction). This is provided by appealing to the null geodesics of the KN and transforming back via Eqs. (4) and (5) to the CX metric. For simplicity we choose $\sigma=0$ in CX (or $\theta=\pi / 2$ in $\mathrm{KN}$ ) to obtain the first integrals of the null geodesics as

$$
\dot{t}=\frac{E\left(r^{2}+a^{2}\right)}{\Delta}, \quad \dot{\phi}=-\frac{a E}{\Delta}, \quad \dot{r}=E
$$

in the KN geometry, and the corresponding first integrals of the CX geometry are

$$
\begin{aligned}
\dot{\tau}= & \frac{E}{m \alpha p}, \quad \dot{y}=\frac{q E}{m \alpha p\left(1-\tau^{2}\right)}, \\
\dot{x}= & -\frac{E}{m^{3} \alpha^{3} p^{2}\left(1-\tau^{2}\right)}\left\{m ^ { 2 } \left[(1-\alpha p \tau)^{2}\right.\right. \\
& \left.\left.+\left(1-\alpha^{2} p^{2}\right)\right]-Q^{2}\right\} .
\end{aligned}
$$

In both cases $E$ is the energy constant and dot represents the appropriate parameter for the null geodesics. We insert two null dust congruences with finite densities $\rho_{l}$ and $\rho_{n}$ propagating along the null vectors $l_{\mu}$ and $n_{\mu}$. In other words the total test energy-momentum tensor is

$$
T_{\mu \nu}=\rho_{l} l_{\mu} l_{\nu}+\rho_{n} n_{\mu} n_{\nu}
$$

where

$$
\begin{array}{r}
l^{\mu}=\left(1,-\frac{\left[(1-\alpha p \tau)^{2}+\alpha^{2} q^{2}\right]}{\alpha^{2} p\left(1-\tau^{2}\right)}, \frac{q}{1-\tau^{2}}, 0\right), \\
n^{\mu}=\left(1, \frac{\left[(1-\alpha p \tau)^{2}+\alpha^{2} q^{2}\right]}{\alpha^{2} p\left(1-\tau^{2}\right)},-\frac{q}{1-\tau^{2}}, 0\right)
\end{array}
$$

in which we have scaled

$$
\frac{E}{m \alpha p}=1 .
$$

The nontrivial scalar $T_{\mu \nu} T^{\mu \nu}$ of the criss-crossing null test dust is given by

$$
T_{\mu \nu} T^{\mu \nu}=8 \rho_{l} \rho_{n} \frac{(1-\alpha p \tau)^{4}}{\alpha^{4}\left(1-\tau^{2}\right)^{2}}
$$

which diverges for $\tau \rightarrow 1$. This corresponds to a divergence for $r \rightarrow r_{-}$in the KN black hole. As a prediction of the HK conjecture any exact back-reaction solution that is imitated by the foregoing test dust must destroy the horizon. In the next section we present a new exact back-reaction solution which represents a solution of colliding Einstein-Maxwellnull dust that exhibits a spacelike singularity for $\tau \rightarrow 1$. The 
new solution incorporates a regular conformal factor (such that it does not diverge as $\tau \rightarrow 1$ ) and therefore leaves all Weyl scalars invariant and regular.

\section{A NEW EXACT BACK-REACTION SOLUTION}

Our aim now is to present an exact solution which involves collision of Einstein-Maxwell fields coupled with null shells. The relations between the mathematical theory of BHs and of colliding waves allows us to find exact solutions which describe the region where two plane waves interact. Then, by a suitable extension it becomes possible to give a complete picture of the incoming space-time before the collision. In this section, we have used this fact to obtain a new solution to the EM fields coupled with null shells. The shells are added as a conformal factor and our method can be summarized as follows.

Let $d s_{0}^{2}$ represent the CX metric (1) which is isometric to the KN. Then, the new metric [12]

$$
d s^{2}=\frac{1}{\phi^{2}} d s_{0}^{2} .
$$

Verification of the metric (13), follows from the substitution of the following into the Ricci scalars (see the Appendix of Ref. [13])

$$
\begin{aligned}
& M=M_{0}+2 \ln \phi, \\
& U=U_{0}+2 \ln \phi, \\
& V=V_{0}, \\
& W=W_{0},
\end{aligned}
$$

where $\left(M_{0}, U_{0}, V_{0}, W_{0}\right)$ correspond to the metric functions of the CX solution and $\phi=1+\alpha_{0} u \theta(u)+\beta_{0} v \theta(v)$, with $\left(\alpha_{0}, \beta_{0}\right)$ positive constants and $\theta$ standing for the step function. Equation (13) represents colliding Einstein-Maxwell fields coupled with null shells. This metric has some advantages over the back-reaction solution of CX. First, while Ricci components and scalar curvature (if any) are affected by inclusion of the conformal factor the Weyl scalars remain invariant (because $M-U=M_{0}-U_{0}$ is the combination that arises in those scalars), i.e., they are finite on the horizon. It turns out as shown in Appendix A explicitly that the scalar curvature and some Ricci components diverge on the horizon. Thus it is misleading to judge the behavior of a horizon by looking only at the Weyl scalars. Our approach gives the clue: it is more reliable to investigate the behavior of the scalar curvature and the Ricci components. In this sense the solution adopted in Eq. (13) as the exact version of the test null dust is stronger (and simpler) than the implication of the source added CX solution [9].

The acceptability of the inclusion of this conformal factor is shown by checking the null and dominant energy conditions of the new solution. The details are given in Appendix B.
The collision of these shells in the background of CX space-time modifies the background in the sense that the curvature scalar, which was zero in the case of $\mathrm{CX}$ is now nonzero and becomes divergent as we approach the horizon. The method employed here should not be confused with the characteristic initial value problem. Extending the solution to the incoming regions to see the waves that participate in the collision is possible.

To find the wave profiles in the incoming regions for example in region II $(u>0, v \leqslant 0)$, we substitute $v=0$ in the obtained metric functions $M, U, V$, and $W$, and using Ricci and curvature scalars given in Appendix A, we obtain

$$
\begin{aligned}
2 \Phi_{22} & =\frac{\alpha^{2}\left(1-\alpha^{2}\right)}{(1-\alpha u)^{4}} \theta(u)+\alpha^{2} \alpha_{0} \delta(u), \\
\Psi_{4} & =\left(\Psi_{4}\right)_{\mathrm{CX}}, \\
\Phi_{00} & =\Phi_{02}=\Phi_{11}=\Lambda=0 .
\end{aligned}
$$

Similarly in region III $(v>0, u \leqslant 0)$, the nonvanishing Ricci and Weyl scalars are

$$
\begin{aligned}
2 \Phi_{00} & =\frac{\alpha^{2}\left(1-\alpha^{2}\right)}{(1-\alpha v)^{4}} \theta(v)+\alpha^{2} \beta_{0} \delta(v), \\
\Psi_{0} & =\left(\Psi_{0}\right)_{\mathrm{CX}}, \\
\Phi_{22} & =\Phi_{02}=\Phi_{11}=\Lambda=0 .
\end{aligned}
$$

The continuity of the metric functions across the null boundaries $u=0, v=0$ makes these scalars continuous across the null boundary too.

As we can see from the incoming waves, in addition to gravitational and electromagnetic fields a matter (shell) field represented by an impulsive component is coupled to the system. Choosing $\alpha_{0}=\beta_{0}=0$ removes the matter field and the resulting solution represented by the metric (13) reduces to CX solution. Since the metric (13) satisfies all these boundary and continuity conditions it must be the correct physical solutions to the present collision problem.

As a second advantage we point out that the metric

$$
d s^{2}=\left(1+\alpha_{0} u+\beta_{0} v\right)^{-2}\left(2 d u d v-d x^{2}-d y^{2}\right)
$$

represents the de Sitter space with scalar curvature and cosmological constant as the only nonzero physical quantities [13]. The transformation

$$
\begin{aligned}
1+\alpha_{0} u+\beta_{0} v & =e^{\lambda t}, \\
\alpha_{0} u-\beta_{0} v & =\lambda z
\end{aligned}
$$

takes this metric into

$$
d s^{2}=d t^{2}-e^{-2 \lambda t}\left(d x^{2}+d y^{2}+d z^{2}\right)
$$

which is identified as the de Sitter metric. Similarly by the choice of the conformal factor and transformation 


$$
\begin{aligned}
1+\alpha_{0} u-\beta_{0} v & =\lambda z, \\
\alpha_{0} u+\beta_{0} v & =\lambda t,
\end{aligned}
$$

our metric becomes

$$
d s^{2}=\frac{1}{\lambda^{2} z^{2}}\left(d t^{2}-d x^{2}-d y^{2}-d z^{2}\right),
$$

which is the anti-de Sitter metric. In both cases the constant $\lambda$ is defined by $\lambda=\sqrt{2 \alpha_{0} \beta_{0}}$ in which $\alpha_{0}>0, \beta_{0}>0$. Now instead of the flat metric by substituting the CX metric (1) it can be interpreted as colliding Einstein-Maxwell fields in a de Sitter background. This is the alternative interpretation that our metric admits when we remove the step functions in the conformal factor. By this interpretation (and of course through the isometry) it says that a $\mathrm{KN} \mathrm{BH}$ endowed with a de Sitter background in between the horizons gives rise to a divergent scalar curvature besides some of the Ricci's. In turn according the HK conjecture such a horizon converts into a singularity. This singularity is necessarily spacelike since a normal vector to the horizon turns out to be timelike. As a final advantage we recall that in the CX metric the null fields are added to the vacuum problem whereas in our case we make the addition to the electrovacuum problem. Such an extension was a missing link in the study of CX.

\section{NULL SINGULARITIES IN THE PRESENCE OF SCALAR FIELDS}

Recently, we have shown the existence of null singularities in the CPW space-time for a class of linearly polarized metrics [14]. Our main objective in this section is to investigate the singularity structure of the $\mathrm{CHs}$ that exist in charged spinning $\mathrm{BHs}$, namely, $\mathrm{KN} \mathrm{BH}$ in the presence of scalar fields. This is achieved through the isometry existing between CPW space-times and BH space-times.

The space-time line element we adopted to describe the collision of plane waves with nonparallel polarization is given by

$$
\begin{aligned}
d s^{2}= & 2 e^{-M} d u d v-e^{-U}\left[\left(e^{V} d x^{2}+e^{-V} d y^{2}\right) \cosh W\right. \\
& -2 \sinh W d x d y] .
\end{aligned}
$$

The complete set of partial differential equations for the metric functions is given elsewhere [15]. The $u, v$ dependent massless scalar field equation

$$
\partial_{\mu}\left(g^{\mu \nu} \sqrt{g} \phi_{\nu}\right)=0
$$

reads as

$$
2 \phi_{u v}=U_{u} \phi_{v}+U_{v} \phi_{u},
$$

where $\phi$ is the scalar field. Given any EM solution we can generate an Einstein-Maxwell-scalar (EMS) solution in accordance with the shift.

$$
M \rightarrow \tilde{M}+\Gamma
$$

where $\tilde{M}$ is any EM solution and

$$
\begin{aligned}
& \Gamma_{u} U_{u}=2 \phi_{u}^{2}, \\
& \Gamma_{v} U_{v}=2 \phi_{v}^{2} .
\end{aligned}
$$

The integrability conditions for the latter equations imply that

$$
\left(\phi_{u} U_{v}-\phi_{v} U_{u}\right)\left(2 \phi_{u v}-U_{u} \phi_{v}-U_{v} \phi_{u}\right)=0 \text {. }
$$

Now any solution of the scalar equation (24) helps us to construct the extra metric function $\Gamma$ by the line integral

$$
\Gamma=2 \int \frac{\phi_{u}^{2}}{U_{u}} d u+2 \int \frac{\phi_{v}^{2}}{U_{v}} d v
$$

As an EM solution, we use the metric (1) obtained by CX which was shown to be locally isometric to the $\mathrm{KN} \mathrm{BH}$ that uses $\tau, \sigma$ coordinates instead of $u, v$.

In terms of $\tau, \sigma$ the scalar field equation (24) and the condition (26) are equivalent to

$$
\left[\left(1-\tau^{2}\right) \phi_{\tau}\right]_{\tau}-\left[\left(1-\sigma^{2}\right) \phi_{\sigma}\right]_{\sigma}=0
$$

and

$$
\begin{aligned}
& \left(\tau^{2}-\sigma^{2}\right) \Gamma_{\tau}=2 \Delta \delta\left(\tau \phi_{\tau}^{2}+\frac{\tau \delta}{\Delta} \phi_{\sigma}^{2}-2 \sigma \phi_{\tau} \phi_{\sigma}\right) \\
& \left(\sigma^{2}-\tau^{2}\right) \Gamma_{\sigma}=2 \Delta \delta\left(\sigma \phi_{\sigma}^{2}+\frac{\sigma \Delta}{\delta} \phi_{\tau}^{2}-2 \tau \phi_{\tau} \phi_{\sigma}\right),
\end{aligned}
$$

respectively. For the present problem we choose the scalar field $\phi$ as

$$
\phi=\frac{k_{1}}{2} \ln \frac{1+\tau}{1-\tau}+\frac{k_{2}}{2} \ln \frac{1+\sigma}{1-\sigma}
$$

in which $k_{1}$ and $k_{2}$ are constant parameters. Using Eqs. (30), the metric function $\Gamma$, due to the scalar field is found as

$$
e^{\Gamma}=\Delta^{-k_{1}^{2}} \delta^{-k_{2}^{2}}(\tau+\sigma)^{\left(k_{1}+k_{2}\right)^{2}}(\tau-\sigma)^{\left(k_{1}-k_{2}\right)^{2}} .
$$

The new metric that describes the collision of EMS fields is expressed by

$$
d s^{2}=X e^{-\Gamma}\left(\frac{d \tau^{2}}{\Delta}-\frac{d \sigma^{2}}{\delta}\right)-\Delta \delta \frac{X}{Y} d y^{2}-\frac{Y}{X}\left(d x-q_{2 e} d y\right)^{2},
$$

where $X, Y, \Delta, \delta$, and $q_{2 e}$ are given in Eq. (2). It is well known that the CX solution is a regular solution. However coupling a scalar field $\phi$ transforms the $\mathrm{CH}$ into a scalar curvature singularity (SCS). This can be seen from the scalar

$$
4 \pi T_{\mu}^{\mu}=\frac{e^{\Gamma}}{\Delta \delta X}\left(\Delta k_{2}^{2}-\delta k_{1}^{2}\right)
$$


and as $\tau \rightarrow 1$ it becomes divergent; hence it is a SCS. The interesting property of this new solution is that the type of the singularity is null rather than spacelike. This can be justified as follows

The singular (or horizon) surface is described by

$$
S(\tau)=1-\tau
$$

(in which we have assumed an equatorial plane, namely, $\sigma$ $=0$, in the transformed BHs space-time). We compute the normal vector to this surface

$$
(\nabla S)^{2}=g^{\tau \tau} S_{\tau}^{2}=\frac{\left(1-\tau^{2}\right) e^{\Gamma}}{X}=\frac{\alpha^{2} \tau^{2\left(k_{1}^{2}+k_{2}^{2}\right)}\left(1-\tau^{2}\right)^{1-k_{1}^{2}}}{(1-p \alpha \tau)^{2}} .
$$

Since we are interested in the limit as $\tau \rightarrow 1$, for $k_{1}^{2}$ $<1,(\nabla S)^{2}=0$ which indicates a null property. The blow-up of the curvature scalar provides a generic null singularity on this surface. The existing isometry can provide us with a null singularity in the corresponding $\mathrm{BH}$ problem.

Using the transformations given in Eqs. (4) and (5) the metric (33) transforms into

$$
\begin{aligned}
\alpha^{2} m^{2} d s^{2}= & \left(1-\rho^{-2} \omega\right) d t^{2} \\
& -\sin ^{2} \theta\left[\Delta+\omega\left(1+a^{2} \rho^{-2} \sin ^{2} \theta\right)\right] d \phi^{2} \\
& -2 a \omega \rho^{-2} \sin ^{2} \theta d t d \phi \\
& -\rho^{2} e^{-\Gamma}\left(\Delta^{-1} d r^{2}+d \theta^{2}\right),
\end{aligned}
$$

where $\rho, \omega$, and $\Delta$ are given in Eq. (6). This metric represents the KN BH coupled with the particular scalar field which reads in the equatorial plane

$$
\phi=\frac{k_{1}}{2} \ln \left(\frac{r_{+}-r}{r-r_{-}}\right) .
$$

In the same plane we have

$$
e^{\Gamma}=\left[\left(r_{+}-r\right)\left(r-r_{-}\right)\right]^{-k_{1}^{2}}\left(\frac{m-r}{\sqrt{m^{2}-Q^{2}}}\right)^{2\left(k_{1}^{2}+k_{2}^{2}\right)} .
$$

Similar analysis on the singular surface

$$
S(r)=r-r_{-}
$$

results in $(\nabla S)^{2}=0$ as $r \rightarrow r_{-}$. This result retains the null singularity formation on the corresponding $\mathrm{BH}$ problem.

Note that, the scalar field in Eq. (31) is singular as $\tau \rightarrow 1$. Let us now choose the following scalar field which is regular, in the limit $\tau \rightarrow 1$,

$$
\phi=\alpha_{0} \tau \sigma,
$$

where $\alpha_{0}$ is any constant parameter. The metric function $\Gamma$ is obtained as

$$
e^{\Gamma}=e^{\alpha_{0}\left[\tau^{2}+\sigma^{2}\left(1-\tau^{2}\right)\right]} .
$$

The curvature scalar due to this scalar field is

$$
4 \pi T_{\mu}^{\mu}=\frac{\alpha_{0}^{2} e^{\Gamma}}{X}\left(\tau^{2} \delta-\sigma^{2} \Delta\right) .
$$

The metric function $\Gamma$ and curvature scalar remains regular as the horizon is approached. The Weyl scalars are also checked and found that as $\tau \rightarrow 1$, the space-time remains regular. This is another justification of the indeterminate character of CHs.

\section{DISCUSSION}

It is known that the relations between the mathematical theory of BHs and that of CPW space-time requires that the interior of every standard $\mathrm{BH}$ solution is locally isometric to the interaction region of CPW space-time.

In this paper, we have used this fact to investigate the singularity structure of the charged spinning BH namely the $\mathrm{KN}$. In our analysis, we have coupled matter (null shells) and scalar fields to the geometry of CPW space-time and using the isometry, we transformed the resulting metric to the $\mathrm{BH}$ geometry and investigated the effect of these fields on the $\mathrm{CH}$. Since our analysis is based on a completely analytic exact solutions, we believe that it reflects the real character of the Killing $\mathrm{CHs}$ of $\mathrm{CPW}$ and $\mathrm{BH}$ space-times.

First, a pair of test null dust is inserted in the CX colliding wave space-time. Since the energy-momentum scalar diverges then we conclude that the $\mathrm{CH}$ is unstable and an exact back reaction solution must yield a scalar curvature singularity. The exact solution which we present involves a regular conformal factor as a source so that all Weyl scalars remain finite while some Ricci's (and scalar curvature) diverge on the horizon. Inclusion of the conformal factor is equivalent energetically to the de Sitter background and through the isometry it makes it possible for us to embed a $\mathrm{BH}$ in a de Sitter (or anti-de Sitter) background. Although we have inserted a matter field as a regular conformal factor, the slightest effect of the source (through the scalar curvature or cosmological constant) of such a background suffices to destroy the inner horizon and converts it into a spacelike curvature singularity. Second, we have inserted scalar fields to the geometry of CX CPW solution. Inclusion of particular scalar fields was shown to create null singularities rather than spacelike ones both in the space of colliding waves and that of corresponding $\mathrm{BH}$ space-times. With these particular exact solutions, we may conclude that the Killing $\mathrm{CH}$ of $\mathrm{CPW}$ space-times and the inner horizon of BHs have dual character when they are subjected to the inclusion of matter and scalar fields. It remains an open question whether this null singularity is an intermediate stage between the horizon and spacelike curvature singularity.

\section{APPENDIX A: THE RICCI AND CURVATURE SCALARS}

The nonzero Ricci and curvature scalars of the collision of Einstein-Maxwell fields coupled with null shells (in the same null tetrad employed by CX) are found as follows: 


$$
\begin{aligned}
\Phi_{22}= & \left(\Phi_{22}\right)_{\mathrm{CX}}+\alpha_{0} \frac{e^{M}}{\phi}\left[\delta(u)+\theta(u) M_{v}\right] \\
\Phi_{00}= & \left(\Phi_{00}\right)_{\mathrm{CX}}+\beta_{0} \frac{e^{M}}{\phi}\left[\delta(v)+\theta(v) M_{u}\right] \\
\Phi_{02}= & \left(\Phi_{02}\right)_{\mathrm{CX}}-\frac{e^{M}}{2 \phi}\left\{\alpha_{0}\left[V_{v} \cosh W-i W_{v}\right] \theta(u)\right. \\
& \left.+\beta_{0}\left[V_{u} \cosh W-i W_{u}\right] \theta(v)\right\}, \\
\Phi_{11}= & \frac{e^{M}}{2 \phi\left(1-u^{2}-v^{2}\right)}\left[\beta_{0} u+\alpha_{0} v\right] \theta(u) \theta(v), \\
\Lambda= & \frac{e^{M}}{12 \phi}\left\{\frac{\left[\beta_{0} u+\alpha_{0} v\right]}{\left(1-u^{2}-v^{2}\right)}+12 \alpha_{0} \beta_{0} \phi^{-1}\right\} \theta(u) \theta(v) .
\end{aligned}
$$

In these expressions $\left(\Phi_{22}\right)_{\mathrm{CX}},\left(\Phi_{00}\right)_{\mathrm{CX}}$, and $\left(\Phi_{02}\right)_{\mathrm{CX}}$ refer to the CX quantities which are all finite on the horizon. Similarly all Weyl scalars are same as in the CX metric, namely regular on the horizon. The other functions $M, V$, and $W$ are given by the following expressions.

$$
\begin{aligned}
e^{V} \cosh W & =\frac{Y}{X \sqrt{\Delta \delta}}, \\
e^{-V} \cosh W & =\frac{X \sqrt{\Delta \delta}}{Y}+\frac{Y q_{2 e}^{2}}{X \sqrt{\Delta \delta}}, \\
e^{-M} & =\frac{2 X}{\sqrt{1-u^{2}} \sqrt{1-v^{2}}}, \\
\phi & =1+\alpha_{0} u \theta(u)+\beta_{0} v \theta(v) .
\end{aligned}
$$

Since $\left(1-u^{2}-v^{2}\right)=\sqrt{1-\tau^{2}} \sqrt{1-\sigma^{2}}$ for $\sigma=0$ and $\tau \rightarrow 1$ (on the horizon) the divergence of the scalar curvature $\Lambda$ and $\Phi_{11}$ is clearly manifest. A detailed calculation reveals that $\Phi_{02}$ is also divergent while $\Phi_{00}$ and $\Phi_{22}$ remain finite on the inner horizon. Let us note that suppressing one of the incoming shells but retaining the other, still the above components diverge. This amounts to colliding Einstein-Maxwell wave from one side with an Einstein-Maxwell-null shell from the other side. However this form can not be interpreted as a de Sitter background in the corresponding black-hole problem.

\section{APPENDIX B: ENERGY CONDITIONS}

It should be noted that the inclusion of the matter field is acceptable if it satisfies some physical conditions such as the null and dominant energy conditions.

\section{Null energy condition (NEC)}

The required condition for the NEC is

$$
T_{\mu \nu} k^{\mu} k^{\nu} \geqslant 0
$$

where $k^{\mu}$ is a null vector tangent to the null geodesics. For the sake of simplicity, we consider the diagonal case of the new solution (13), that represents null shells in the RN geometry. The null tetrads are

$$
\begin{aligned}
& l^{\mu}=\frac{1}{\sqrt{2}}\left(\frac{\alpha}{1-\alpha \tau}, \frac{\alpha}{1-\alpha \tau}, 0,0\right) \\
& n^{\mu}=\frac{1}{\sqrt{2}}\left(\frac{\alpha}{1-\alpha \tau},-\frac{\alpha}{1-\alpha \tau}, 0,0\right), \\
& m^{\mu}=-\frac{1}{\sqrt{2}}\left(0,0,-\frac{(1-\alpha \tau)}{\alpha \sqrt{\Delta}}, \frac{i \alpha}{\sqrt{\delta}(1-\alpha \tau)}\right), \\
& \bar{m}^{\mu}=-\frac{1}{\sqrt{2}}\left(0,0,-\frac{(1-\alpha \tau)}{\alpha \sqrt{\Delta}},-\frac{i \alpha}{\sqrt{\delta}(1-\alpha \tau)}\right) .
\end{aligned}
$$

It is found that

$$
\begin{gathered}
T_{\mu \nu} l^{\mu} l^{\nu}=\Phi_{00}, \\
T_{\mu \nu} n^{\mu} n^{\nu}=\Phi_{22},
\end{gathered}
$$

where

$$
\begin{gathered}
\Phi_{00}=\frac{\alpha^{2}\left(1-\alpha^{2}\right)}{2(1-\alpha \tau)^{4}}+\frac{\alpha e^{M}}{\phi}\left[\delta(u)+M_{v} \theta(u)\right], \\
\Phi_{22}=\frac{\alpha^{2}\left(1-\alpha^{2}\right)}{2(1-\alpha \tau)^{4}}+\frac{\beta e^{M}}{\phi}\left[\delta(v)+M_{u} \theta(v)\right],
\end{gathered}
$$

which are positive and therefore satisfy NEC.

\section{Dominant energy condition (DEC)}

The DEC is defined as

$$
T^{00} \geqslant\left|T^{a b}\right|
$$

i.e., for each $a, b$, in the orthonormal basis the energy dominates the other component $T_{a b}$. The orthonormal vectors for the diagonal case of the new solution are

$$
\begin{aligned}
& e_{(0)}^{\mu}=\left(\frac{1}{\sqrt{X}}, 0,0,0\right), \\
& e_{(1)}^{\mu}=\left(0,-\frac{1}{\sqrt{X}}, 0,0\right),
\end{aligned}
$$




$$
\begin{aligned}
& e_{(2)}^{\mu}=\left(0,0, \frac{1-\alpha \tau}{\alpha \sqrt{\Delta}}, 0\right), \\
& e_{(3)}^{\mu}=\left(0,0,0,-\frac{\alpha}{\sqrt{\delta}(1-\alpha \tau)}\right) .
\end{aligned}
$$

The nonzero energy-momentum tensors in orthonormal frames are

$$
\begin{aligned}
& T_{00}=\frac{1}{8 \pi}\left[\Phi_{00}+\Phi_{22}+2\left(\Phi_{11}+3 \Lambda\right)\right], \\
& T_{01}=T_{10}=\frac{1}{8 \pi}\left[\Phi_{22}-\Phi_{00}\right],
\end{aligned}
$$

where the expressions for $\Phi_{00}, \Phi_{22}, \Phi_{11}, \Phi_{02}$, and $\Lambda$ are
[1] S. Chandrasekhar and B.C. Xanthopoulos, Proc. R. Soc. London A415, 329 (1988).

[2] U. Yurtsever, Phys. Rev. D 36, 1662 (1987).

[3] P. Bell and P. Szekeres, Gen. Relativ. Gravit. 5, 275 (1974).

[4] Y. Gursel, V.D. Sandberg, I.D. Novikov, and A.A. Starobinsky, Phys. Rev. D 19, 413 (1979); S. Chandrasekhar and J.B. Hartle, Proc. R. Soc. London A284, 301 (1982).

[5] A. Ori, Phys. Rev. Lett. 68, 2117 (1992).

[6] L. Burko, Phys. Rev. Lett. 79, 4958 (1997).

[7] L. Burko, Phys. Rev. D 59, 024011 (1998).

[8] U. Yurtsever, Class. Quantum Grav. 10, L17 (1993). given in Appendix A.

$$
\begin{aligned}
& T_{11}=\frac{1}{8 \pi}\left[\Phi_{00}+\Phi_{22}-2\left(\Phi_{11}+3 \Lambda\right)\right], \\
& T_{22}=\frac{1}{4 \pi}\left[\Phi_{00}+\Phi_{11}-3 \Lambda\right],
\end{aligned}
$$$$
T_{33}=\frac{1}{4 \pi}\left[\Phi_{11}-\Phi_{02}-3 \Lambda\right],
$$

[9] S. Chandrasekhar and B.C. Xanthopoulos, Proc. R. Soc. London A414, 1 (1987).

[10] S. Chandrasekhar and B.C. Xanthopoulos, Proc. R. Soc. London A418, 175 (1986).

[11] T.M. Helliwell and D.A. Konkowski, Class. Quantum Grav. 16, 2709 (1999).

[12] M. Halilsoy, J. Math. Phys. 41, 8351 (2000).

[13] A. Wang, J. Math. Phys. 33, 1065 (1992).

[14] O. Gurtug and M. Halilsoy, gr-qc/0103092.

[15] O. Gurtug and M. Halilsoy, gr-qc/0006038. 